\title{
Is Knowledge Enough? Considering HIV|AIDS Risk Behaviors and HIV/AIDS Knowledge with African American Women
}

\author{
Emory L Perkins ${ }^{1,{ }^{*}}$; Kesslyn Brade Stennis ${ }^{1}$; Velva Taylor Spriggs ${ }^{1}$; Emma Aya Kwegyir-Afful ${ }^{1}$; \\ Aaron Prather ${ }^{1}$ \\ ${ }^{1}$ Department of Social Work, Bowie State University, Bowie, USA \\ *Corresponding author: Emory L Perkins, Department of Social Work, Bowie State University, Bowie, USA. Tell: +301-8603272, Fax:+301-8603163, E-mail: eperkins@bowiestate.edu
}

Received: September 25, 2013; Revised: January 7, 2014; Accepted: June 2, 2014

\begin{abstract}
Background:This quantitative explanatory study was designed to explain the relationship between HIV/AIDS risk-taking behaviors among African American women and their knowledge surrounding HIV/AIDS. More specifically, the research considers whether knowledge alone was sufficient to alter African-American women's risk-taking behaviors regarding HIV|AIDS.

Objectives: This study was designed to investigate knowledge surrounding sexual risk-taking behaviors including 1) heterosexual transmission; 2) low and sporadic condom usage; 3) illicit drug use; and 4) multiple sex partners associated with HIV positive and negative status among African-American women.

Patients and Methods: Participants in this study were African-American women who ranged in age between 24 and 44 years. This sample of African-American women was divided into two groups according to their HIV status: 53 women who were HIV positive and 62 women who were HIV negative women. The data for this investigation were obtained through self-administered questionnaires. The t-test was used to analyze the data through the use of the windows version of the statistical package for social sciences (19.0).

Results: Findings suggest that knowledge of HIV was not found to be significantly different for the HIV positive and negative groups, and thus, suggest that knowing about the consequences of risk-taking behaviors is not sufficient to alter one's behavior among African American women. The findings from this investigation will contribute new empirical knowledge to the existing social work database on HIV/AIDS and knowledge specifically relative to African-American women.

Conclusions: It was anticipated that knowledge of HIV would have been significantly higher in the HIV negative group than in the HIV positive group. However, the hypothesis was not supported by data. The HIV positive women and negative women were equally knowledgeable about HIV/AIDS.
\end{abstract}

Keywords: HIV/AIDS; Acquired Immunodeficiency Syndrome; Knowledge; Risk-Taking; African Americans; Women

\section{Background}

Now in the beginning of its fourth decade, acquired immune deficiency syndrome(AIDS) continues to be a public health crisis. According to the Centers for Disease Control there have been more than 1,178, 350 people aged 13 or older living with AIDS in the United States. It is also estimated that there are more than 12 millions people in the United States living with HIV infection, and 1 in 5 is unaware of the infection (1). The World Health Organization (WHO) maintains that an estimated 33.3 million people are living with HIV/AIDS in the Americas (i.e. United States, Canada, Latin America and the Caribbean) and more than 35.9 millions people worldwide are living with the disease. The statistics also reveal that since the beginning of the epidemic, about 60 millions people have been infected with the HIV virus and about 25 millions have died (2).

The African-American community continues to be disproportionately affected by HIV/AIDS. In 2009, African Americans comprised $14 \%$ of the United States population but accounted for $44 \%$ of all new HIV infection (3). Among African-American men, women and adolescents, there have been more than 466,351 diagnosed cases of AIDS in the United States (3). According to the CDC (3, 4 ), AIDS is now the leading cause of death for AfricanAmerican women aged 25-34. Further, African-American women are 20 times more likely to become infected with HIV compared to their white female counterparts. Conversely, young people aged 24 to 44 .

Women of color are disproportionately impacted by HIV infections in the United States. It is estimated that about $15 \%$ of women who are HIV positive are unaware of their HIV status. Centers for Disease Control asserts that $84 \%$ of the HIV infections that occurred were from heterosexual contact, accounting for the fourth largest number of all new HIV infections among all people in the United States including Black/African American women with heterosexual contact $(3,4)$. While there are a number of factors that impact HIV transmission, those being addressed as factors that directly and indirectly impact the increased incidences associated with the risk-taking behaviors among African-American women are 1) hetero-

Copyright @ 2014, Zahedan University of Medical Sciences; Published by Kowsar Corp. This is an open-access article distributed under the terms of the Creative Commons Attribution License, which permits unrestricted use, distribution, and reproduction in any medium, provided the original work is properly cited. 
sexual transmission; 2) low and sporadic condom usage; 3) illicit drug use; and 4) multiple sex partners.

\subsection{Heterosexual Transmission}

Many African-American women have reportedly contracted HIV from a male partner who has injected drugs, had multiple sex partners, and/or have been living on the "down low" (DL) or as a "man having sex with men" (MSM). These last two terms generally refer to a man who self-identifies as heterosexual and maintains a sexual and emotional relationship with women while having discrete sexual relations with men (4-6). African-American men represented almost one-third of all HIV infections in 2009 and accounted for 70 percent of new infections among blacks (3). Additionally, the Centers for Disease Control maintains that men who have sex with men represent the largest proportion of new infections, an estimated $61 \%$ of the reported HIV cases in 2009 , subsequently infecting a disproportionate number AfricanAmerican women with HIV and other STIs/STDs in part because they are unaware of their partner's DL/MSM status $(3,7)$. Millett et al. $(6,8)$ maintain that "men on the down-low" have been cited as the fundamental reason for the disproportionate increase in HIV/AIDS among African-American women.

\subsection{Low and Sporadic Condom Usage}

In the African-American community, limited condom usage is also linked to HIV/AIDS. One reason is that condom usage is often not negotiated because of women's fear of reprisal, inconvenience and negative perceptions about condom use on the part of men $(7,9)$. Another is the perception that HIV infection is a "white gay disease" and does not impact heterosexuals, particularly those in a monogamous relationship, as they believe themselves to be. Therefore, there is no perceived need to wear a condom for the purpose of protecting oneself from HIV/AIDS (10).

\subsection{Use of Illicit Drugs}

Prior to 1994, the CDCP (Centers for Disease Control and Prevention) noted that illicit drug use was the foremost cause of HIV transmission in the United States for AfricanAmerican women. It has been reported that about $19 \%$ of all AIDS cases in 2003 were acquired through intravenous drug use. Currently, about $8 \%$ of all the new HIV infections are among intravenous drugs abusers in the United States. Unfortunately, African-American women and men accounted for a significant number of the new HIV infections among intravenous drug users $(3,4,9)$.

\subsection{Multiple Sex-Partners and HIV/AIDS}

According to the $\mathrm{CDC}$ reports $(3,4)$, having multiple sex-partners is another factor that impacts HIV infection rates. According to a study conducted by AIDS Research
Institute (4) many African-American women reported having multiple sex partners due to socio-economic factors and societal structural issues. It was further maintained that a large segment of these African-American women was not able to successfully negotiate condom use as they felt that it would interfere with physical and emotional intimacy, and subsequently, their male partners would suspect them of having sexual intercourse with other male partners. Another research study conducted by CDCP (Center for Disease Control and prevention, in conjunction with the North Carolina Department of Health $(\mathrm{NCDOH})$ documented that the epic proportion of HIV infection among African-American women in North Carolina was related to multiple sex partners. According to the findings, a large majority of negative and positive sexually active African-American women reported that they engaged in sexual behaviors that placed them at increased risk for HIV infection, including having multiple sex partners, not successfully negotiating condom use, and their perception that they were engaging in sex with men who were not HIV infected $(4,11)$.

In summary, the literature reports that a large majority of African-American women continue to be infected with HIV/AIDS through heterosexual transmission, the sporadic use of condoms, the use of illicit drugs by themselves or their partners, and having multiple sex partners who may or may not reveal their HIV/AIDS status. What is not discussed in the literature is the impact of specific factors like knowledge surrounding HIV/AIDS and related risk taking behaviors of African American women.

\section{Objectives}

This study was designed to investigate knowledge surrounding sexual risk-taking behaviors including 1 ) heterosexual transmission; 2) low and sporadic condom usage; 3) illicit drug use; and 4) multiple sex partners associated with HIV positive and negative status among African-American women.

\section{Patients and Methods}

A quantitative, exploratory study was utilized to examine whether knowledge of HIV/AIDS alone is sufficient to alter African-American women's HIV/AIDS related risktaking behaviors. In this study one hypothesis was tested. Using an ex post facto research design, the researcher surveyed two randomly selected groups of African American women based on their existing HIV status, namely positive women confirmed by self-disclosure and staff report and negative women determined by self disclosure and observation. There was no opportunity to manipulate HIV status, knowledge of HIV or risk-taking behaviors, as these were merely assessed after-the-fact.

\subsection{Instruments}

In addition to nine demographic items that ascertained age, race, education attainment, employment sta- 
Perkins EL et al.

tus, income, and source of income, sexual orientation, number of children, residence and HIV status, The HIV risk-taking behavior scale (HRBS), the AIDS knowledge questionnaire (HIV-K-Q), and the demographic questionnaires were used to collect the data. The instruments covered specific questions about:1) beliefs in condom usage; 2 ) number of sexual partners within the last month;3) types of risky behaviors in which they participated; 4) how often you used condoms while engaging in sex with their regular partner(s) in the last month; 5 ) number of times you had anal sex in the last month; and 6) the number of different people with whom you shared a needle within the last month. The eleven items on the HRBS were tested by Ward et al. (12), for test-retest reliability (one week), yielding a Pearson coefficient of .86 and validity. Cronbach alpha's of .70 for a group with potential risk for HIV/AIDS. The test sample included 175 adults (ages 18-42) from Australia. This scale has not been used with African-American women at risk for becoming infected with HIV/AIDS. However, the HIV knowledge questionnaire authored by Carey et al. (13) was developed and judged to be a reliable, valid and practical, self-administered questionnaire 0.75 to 0.89 internal consistencies.

\subsection{Sampling Procedures}

IRB (institutional review board) approval was granted by a university to conduct a non probability (purposive) sample used to identify African-American women ages 24 to 54. This group was equally divided into two groups of women, HIV positive and HIV negative status. The group of self-identified HIV positive women was recruited from a local addiction agency. The purpose of the study was briefly discussed with the potential participants, with the assistance of a staff minister, a second group of African-
American women were recruited from a Baptist church. The researcher briefly discussed the purpose and significance of the study, and subjects were informed that their participation was voluntary and confidential. Over a two-week period, all participants provided consent to participate in the study, the participants completed an alphanumerically-coded questionnaire and, subsequently, were given a twenty-five dollar gift certificate for their participation.

\section{Results}

The research hypothesized that knowledge of HIV will be significantly higher in the HIV negative group than in the HIV positive group. To address this hypothesis, the relationship between knowledge of HIV status was tested utilizing a t-test.

The t-test in Table 2 shows that there was no significant difference between the mean score for the HIV knowledge positive and negative participants. This was anticipated, but was not found to be the case, while the mean knowledge score for HIV positive group was 41.43, compared to the 40.08 for the HIV negative group, this small difference was not statistically significant $(t=95.645$, df $=-1.091$ and $\mathrm{P}=0.278$ ). Therefore, hypothesis $\mathrm{H}: 1$ knowledge of HIV will be significantly higher in the HIV positive group than in the HIV negative group, which is not supported by the data. The researcher had predicted that those with greater knowledge would less likely become victims of the HIV infection.

It was anticipated that knowledge of HIV would have been significantly higher in the HIV negative group than in the HIV positive group. However, this hypothesis was not supported by data. The HIV positive women and negative women were equally knowledgeable about HIV/AIDS.

Table 1. t-test on HIV Status by Knowledge of HIV

\begin{tabular}{lcccccc}
\hline HIV status & Number & Mean \pm SD & Se & t & df & PValue \\
\hline Positive & 52 & $40.08 \pm 6.642$ & 0.921 & -1.091 & 95.645 & 0.278 \\
Negative & 60 & $41.43 \pm 5.157$ & 0.666 & & & \\
\hline
\end{tabular}

Table 2. Summary of Data

\begin{tabular}{lccc}
\hline Hypothesis & t-test & P Value & Conclusion \\
\hline $\begin{array}{l}\text { H:1 Knowledge of HIV Will Be Significantly Higher in the HIV Positive } \\
\text { Group than in the HIV Negative Group }\end{array}$ & -1.091 & 0.278 & not supported \\
\hline
\end{tabular}

\section{Discussion}

The scope and magnitude of the HIV/AIDS phenomenon paint a rather dismal picture. The existence of this epidemic calls for more theory application, practice ventures and research that consider the etiology, prevention and treatment of HIV/AIDS. More specifically, activism within and on behalf of HIV/AIDS impacted communities and populations must include an awareness that AfricanAmerican women are disproportionately infected, and 
that unique prevention and intervention approaches must be conceptualized and implemented. If AfricanAmerican women are going to be protected from HIV/AIDS infection, society's cultural and structural issues must be addressed by going beyond "knowledge and awareness" and including skill mastery and practice approaches that consider, cultural beliefs, health care, access, prevention, early intervention and treatment, which are tailored to meet the needs of this specific population.

\subsection{Theory}

Social cognitive theory as proffered by Bandura postulates that knowledge alone is not sufficient to explain one's behavior, which was confirmed through this study. Bandura et al. (14) asserted that in order to change one's behavior, there are several essential constructs that must be infused into the decision-making process relative to participation in a behavior. Based on Bandura's Social Cognitive Theory, persons working with and advocating on behalf of HIV/AIDS infected African American women must include components that encompass several considerations. First, individuals must have increased awareness and knowledge of safe-sex practices, intravenous drug use implications and vulnerabilities. Additionally, those engaging in HIV-related behaviors must have selfefficacy in negotiating safer sex practices and condom usage. Third, individuals must acquire the necessary prerequisite skills for self-control and risk-reduction strategies to prevent risk-taking behaviors. Fourth, promoting efficacy within individuals should assist them with making decisions about how to negotiate condom usage and safer sex practice. Finally, if individuals are going to maintain the newly acquired skills, there needs to be social support upon which the individual rely.

\subsection{Practice}

Contrary to what was hypothesized, this study reveals that knowledge about HIV/AIDS is not enough to impact practice behaviors associated with contracting HIV/ AIDS. While the study does not negate the importance of knowledge, it simply confirms that knowledge alone does not have a significant relationship with HIV/AIDS related risk-behaviors. Therefore, practitioners must expand their ideas about HIV/AIDS awareness to include components beyond increasing knowledge.

One recommendation is for practitioners to include skill development components in their training programs. Those practicing HIV risk-related behavior should have skills development programs that address condom negotiation, safe-sex practices and HIV-testing. Additionally, health promotion programs must be infused into practice models and prevention/intervention strategies which must target all African-Americans, specifically African-American women. Furthermore, culturally sensitive intervention programs must be developed to address the disproportionate number of HIV/AIDS cases among Afri-
can-Americans. Such models should not only teach African-American women negotiation skills, but the models must include a practice component which will empower them to demonstrate negotiation skills surrounding condom use and other safe sex practices.

The interactive, skill-based model must cover re-infection as a major concern. African-Americans must be informed that re-infection may cause an individual's immune system to become further compromised due to the emergence of new and different strains of the HIV virus. A logistic model of health promotion must be addressed in the forms of educational, prevention and interventional programs. The new model of interventions must make a formidable attack on the usual knowledge-based methods and must include alternative approaches.

\subsection{Research}

The findings from this investigation contribute new empirical knowledge to the existing social work database in that they document the experiences of African-American women surrounding knowledge as a deterrent to risktaking behavior. However, the findings suggest that there is a great void in the literature and that further research must be conducted.

While the current study addressed a special sub-cultural group, namely African-American women, more in-depth empirical research is needed which expands HIV/AIDS knowledge to include this population and those professionals with whom they have contact. Such research must include service providers, educators, researchers, public health officials, policy makers and African-American women including the development of effective plans that include additional prevention and intervention components, which supplement the HIV/AIDS knowledge base. Regional and national studies are needed to explore the multi-faceted nature of this pandemic as a national problem as well as the multi-faceted approaches needed to address the pandemic. Topical areas that warrant research are numerous. Priorities may be socioeconomic factors and special areas of vulnerability for certain subcultural groups, such as women, seniors and persons living with disabilities. In summary, the researchers must develop, review, test and enhance culturally-sensitive models which focus on target populations that interface with African American women engaged in risk-taking behavior.

\section{Acknowledgements}

The authors acknowledge those African American women who participated in this study and the community based organizations.

\section{References}

1. HIV Surveillance report atlanta. USA: Centers for Disease Control and Prevention Diagnoses of HIV infection in the United States and Dependent Areas; 2013.

2. World Health Organization WHO. AIDS epidemic update: Women 
and AIDS; 2009.

3. Centers for Disease Control and Prevention. National Center for HIV, STD, and TB Prevention, Division of HIV/AIDS Prevention; 2014.

4. HIV Surveillance Report Atlanta: CDC: Centers for Disease Control and Prevention Diagnoses of HIV infection in the United States and Dependent Areas; 2013.

5. Sharpe TT. Sex-for-crack-cocaine exchange, poor black women, and pregnancy. Qual Health Res. 2001;11(5):612-30.

6. Whyte J, Whyte MD, Cormier E. Down low sex, older African American women, and HIV infection. J Assoc Nurses AIDS Care. 2008;19(6):423-31.

7. Valleroy L, A, Montgomery C, Mokotoff DH, Gentry C. HIV prevalence and associated risks in young men who have sex with men. J Am Med Assoc. 2002;284(37):198-204.

8. Millett G, Malebranche D, Mason B, Spikes P. Focusing "down low" bisexual black men, HIV risk and heterosexual transmission. J Natl Med Assoc. 2005;97(7):525-95.

9. Timpson S, C, Williams ML, Bowen AM, Atkinson JS, Ross MW.
Sexual activity in HIV-positive African American women crack cocaine smokers. Arch Sex Behav. 2010;39(6):1353-8.

10. Mays VM, Cochran SD. Acquired immunodeficiency syndrome and black Americans: special psychosocial issues. Public Health Rep.1987;102(2):224.

11. North Carolina Department of Health and Human Services. Epidemiologic profile for 2005 HIV/STD prevention and care planning. North Carolina Department of Health and Human Services; 2004

12. Ward J, Darke S, Hall W. The HIV risk-taking behaviour scale (HRBS) manual: National Drug and Alcohol Research Centre, University of New South Wales Sydney; 1990.

13. Carey MP, Morrison-Beedy D, Johnson BT. The HIV-Knowledge Questionnaire: Development and evaluation of a reliable, valid, and practical self-administered questionnaire. AIDS Behav. 1997;1(1):61-74.

14. Bandura A, Jourden FJ. Self-regulatory mechanisms governing the impact of social comparison on complex decision making. JPers Soc Psychol.1991;60(6):941. 